\title{
Joint Admission Control and Association for the Downlink of a mmWave Vehicular Network
}

\author{
Akila Ekanayake, K. B. Shashika Manosha, Nandana Rajatheva, and Matti Latva-aho \\ Centre for Wireless Communications, \\ University of Oulu, \\ Finland \\ E-mail: akila.ekanayake@ nokia.com, \{manosha.kapuruhamybadalge, nandana.rajatheva, matti.latva-aho\}@oulu.fi
}

\begin{abstract}
In this study, we consider the problem of admission control for primary vehicles and association for secondary vehicles in a singe cell downlink vehicular network, based on vehicle-to-everything (V2X) architecture. We consider the network operates on millimeter wave (mmWave) communication at $60 \mathrm{GHz}$. Our objective is to determine the optimal topology of vehicle connectivity which maximizes the number of admitted primary vehicles, while associating all secondary vehicles to ensure the coverage. We cast the optimization problem as an $\ell_{0}$ minimization problem. This problem is known to be combinatorial and NP-hard. Hence, we propose a sub-optimal, two-stage algorithm to solve it. With numerical results we show that the proposed algorithm achieves close to optimal performance.

Index Terms-Admission control, Vehicle association, Vehicular networks, $\ell_{0}$ minimization, mmWave.
\end{abstract}

\section{INTRODUCTION}

Due to new socio-economic trends, transportation is evolving in a rapid phase. New urbanization wave demands the development of existing transportation infrastructure, while social trends such as environmental regulations, increasing demand for improved public transport services are transforming the requirements of transportation industry. Development of automated driving, new vehicle ownership models and information availability are economic trends which shift current transportation systems towards intelligent transportation systems (ITS) [1]. Vehicle-to-Everything (V2X) communication is a key integral component of ITS, which involves wireless connectivity between the vehicle and its surrounding environment. With the advancement of technology, vehicles are becoming more intelligent and less reliant on human interaction. Hence, V2X communication is dynamic and involves many systems and subsystems [2].

V2X standard developed by 3GPP in release 14 is referred as cellular V2X or LTE-V. It mainly aims to utilize the existing cellular communications infrastructure to support V2X services. Since infrastructure elements are not always reliable, direct communication is needed between V2X elements. In the same release, $3 \mathrm{GPP}$ introduced mode three and mode four to support new use cases in V2X communication. In mode three, radio resources are managed by the cellular network and in mode four vehicles manage the radio resources autonomously.

This research was supported by the Academy of Finland 6Genesis Flagship under Grant 318927.
Since traffic safety related V2X services cannot rely on the cellular network, mode four is considered as the baseline vehicle-to-vehicle (V2V) transmission mode [3], [4].

In literature for vehicular ad-hoc networks (VANETs), V2V communication is considered as the traditional protocol for short range vehicular networks. However, VANETs are challenging due to dynamic network topology changes, frequent discontinuities in communication links due to blockages and limitations of communication ranges, highly mobile nature of vehicle movements and sparse distribution of vehicles [5], [6]. Existing work has been done extensively on vehicle-toinfastructure (V2I) and V2V communications for delivering ITS use cases. In order to deliver the challenging requirements, research focus has been shifted towards employing joint V2V and V2I communications. According to [7], [8] joint $\mathrm{V} 2 \mathrm{~V}$ and $\mathrm{V} 2 \mathrm{I}$ architecture outperforms individual performance of V2I and V2V communications.

Admission control can be used in situations where all the users cannot be served in the system. In VANETs when vehicle density is high, base station (BS) or road side unit (RSU) might not be able to serve all the vehicles. Admission control can be employed in V2I scenarios to select the optimum set of vehicles to establish connectivity. As shown in [9] user admission problems are formulated as optimization problems, but they can easily become infeasible. In such scenarios constraints of the optimization problems have to be relaxed by either decreasing the target signal-to-interferenceplus-noise-ratio (SINR) values, or by changing the number of users if there exists no feasible solution to the optimization problem [10]. In [11] admission control problem is formulated as an $l_{0}$ minimization problem for multi-cell downlink system. Also an exhaustive search algorithm can be proposed as a solution, where for a given set of admitted users, users are added until the problem becomes infeasible [9]. In the joint V2V and V2I architecture, V2V communication is used to extend the communication for vehicles which are out of coverage from the BS. These vehicles can be associated with vehicles which have already established a V2I link. Device-todevice communication based user association has been widely adopted as in [12], [13] for V2X applications.

mmWave communication is becoming a promising technology to support challenging high throughput use cases of 
5G vehicular communications such as intelligent driving, infotainment and extended sensors. In vehicular networks there are several key challenges in implementing mmWave communication. One of the main challenges is mmWave requires a line-of-sight between the transmitter and the receiver. Due to buildings, pedestrians and other vehicles line-of-sight can be disturbed. To overcome pathloss a directional transmission technology specifically directional antenna or beamforming is required [14] [15].

In this study we propose an algorithm to optimize the joint admission and association, for joint V2V and V2I architecture based on mmWave communication at $60 \mathrm{GHz}$. To the best of our knowledge, in previous studies admission control and association have been used in vehicular networks separately, but no previous work have been done using joint admission control and association for joint $\mathrm{V} 2 \mathrm{~V}$ and V2I architecture to determine the optimal connectivity. We propose a sub-optimal, two-stage algorithm to solve this problem. This algorithm maximizes the number of primary vehicles while associating all secondary vehicles to admitted primary vehicles, thus it ensures the reliable connectivity from BS to out of range vehicles.

The paper is organized as follows. In Section II system model is described. Problem formulation is presented in Section III and in Section IV we present the proposed algorithm. Numerical results are provided in Section V. Finally Section VI concludes the paper.

\section{SySTEM MODEL}

We consider a network with a single base station and set of vehicles. We denote the set of all vehicles by $\mathcal{V}$ and base station or road side unit by $b$. We define primary vehicles as vehicles which can directly communicate with BS $b$ and secondary vehicles as vehicles which cannot directly communicate with BS, but able to directly communicate with primary vehicles as illustrated in Fig. 1.

We denote the set of all primary vehicles by $\mathcal{P}$ and we label them with the integer $i=1, \ldots, I$. The set of all secondary vehicles are denoted by $\mathcal{S}$ and we label them with the integer $j=1, \ldots, J$.

Standard log-distance pathloss model in [16] is adopted to model $60 \mathrm{GHz}$ mmWave channel and to account for blockage effects. In this model the channel gain for link $L_{i j}$ between primary vehicle $i$ and secondary vehicle $j$ is given by

$$
g_{i j}^{c}=10 \delta_{i j} \log \left(d_{i j}\right)+15 d_{i j} / 1000+\beta_{i j},
$$

where $d_{i j}$ is the distance between primary vehicle $i$ and secondary vehicle $j$, and $\delta_{i j}$ is the path loss exponent. The second term represents the atmospheric attenuation at $60 \mathrm{GHz}$, and third term $\beta_{i j}$ depends on the number of blockers that obstruct the link $L_{i j}$. Similar equations for channel gain can be derived for links between primary vehicles to BS $b$.

Sectored antenna model [17] is adopted to approximate the beamforming antenna patterns. In this antenna model, the gain is a constant for all angles in the main lobe and the gain is

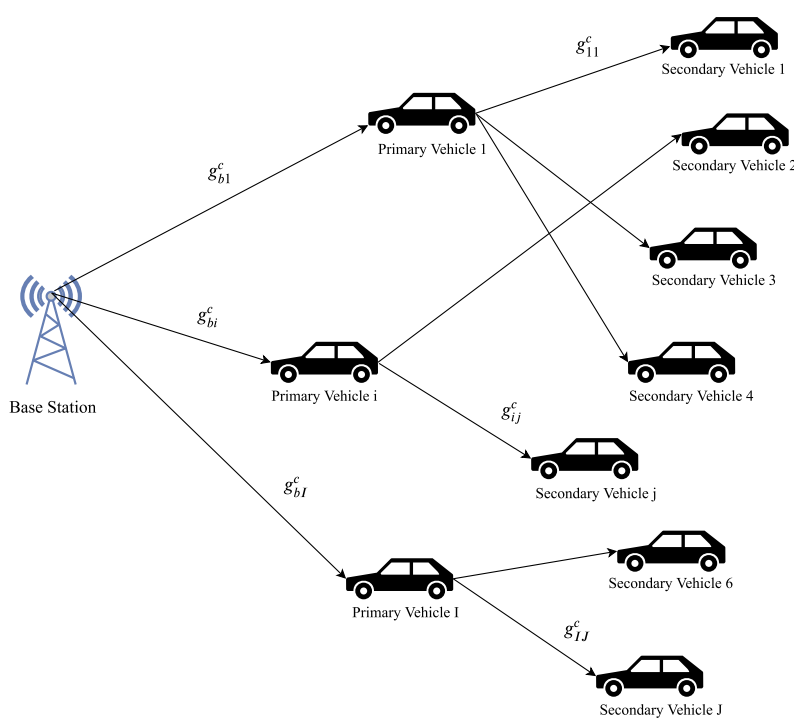

Fig. 1. System model

a smaller constant in the side lobe. Transmitter gain $g_{i j}^{t}$ for link $L_{i j}$ is represented by

$$
g_{i j}^{t}= \begin{cases}\frac{2 \pi-\left(2 \pi-\varphi_{i j}^{t}\right) z}{\varphi_{i j}^{t}}, & \text { if }\left|\theta_{i j}^{t}\right| \leq \varphi_{i j}^{t} / 2 \\ z, & \text { otherwise, }\end{cases}
$$

where $\theta_{i j}^{t}$ represents the alignment error between primary vehicle $i$ and secondary vehicle $j$ th antenna steering direction and the corresponding boresight direction. We represent halfpower-beamwidth of the of link $L_{i j}$ at transmission side by $\varphi_{i j}^{t}$. Using a similar approach one can obtain receiver gain $g_{i j}^{r}$ for link $L_{i j}$. Non-negligible sidelobe power is represented by $z$, where $0 \leq z \leq 1$. Equations for the gains $g_{b i}^{t}$ and $g_{b i}^{r}$ for the link $L_{b i}$ can be written in a similar way.

The total bandwidth allocated for BS to primary vehicle communication is $B_{b p}$ and the total bandwidth allocated to primary to secondary vehicle communication is $B_{p s}$. Furthermore, we assume that these two bandwidth portions are orthogonal as illustrated in Fig. 2, and hence there is no interference from BS-primary communication to primarysecondary communications, and vice-versa.

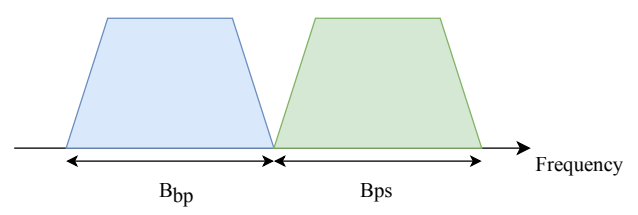

Fig. 2. Orthogonal bandwidth allocation for $B_{p s}$ and $B_{b p}$.

The received SINR of $i$ th primary vehicle of $\mathrm{BS} b$ is given by

$$
\Gamma_{b i}=\frac{P_{b i} g_{b i}^{r} g_{b i}^{c} g_{b i}^{t}}{\sum_{l \in \mathcal{P}, l \neq i} P_{b l} g_{b i}^{r} g_{b i}^{c} g_{b i}^{t}+B_{b p} N_{0}},
$$


where Gaussian noise power density is denoted by $N_{0}$.

Let $X^{s}$ be the primary-secondary link association matrix, where $(i, j)$ th element is represented by

$$
x_{i j}= \begin{cases}1, & \text { if link } L_{i j} \text { is established } \\ 0, & \text { otherwise }\end{cases}
$$

which indicates whether link $L_{i j}$ is established or not. The received SINR of $j$ th secondary vehicle is given by

$$
\Gamma_{i j}=\frac{x_{i j} P_{i j} g_{i j}^{r} g_{i j}^{c} g_{i j}^{t}}{\sum_{l \in \mathcal{P}, l \neq i} P_{l j} g_{l j}^{r} g_{l j}^{c} g_{l j}^{t}+B_{p s} N_{0}} .
$$

To guarantee the quality of service of all links, SINR levels should satisfy the below inequalities

$$
\begin{aligned}
& \Gamma_{b i} \geq \Gamma_{b i}^{t h}, i \in \mathcal{P}, \\
& \Gamma_{i j} \geq \Gamma_{i j}^{t h}, j \in \mathcal{S} .
\end{aligned}
$$

\section{PROBlem Formulation}

Our goal is to maximize the number of admitted primary vehicles and associate of all secondary vehicles to the admitted primary vehicles, while satisfying the system constraints. We now formulate this design problem as a mathematical optimization problem. To do this, we introduce the nonnegative auxiliary variables for link admission between BS and primary vehicles.

$$
\Gamma_{b i} \geq \Gamma_{b i}^{t h}-a_{b i}, \forall i \in \mathcal{P}
$$

In (8), when $a_{b i}=0$ we recover constraint (6), i.e., the SINR constraint of $i$ th primary vehicle is satisfied. Furthermore, by making $a_{b i}$ large enough the set of relaxed SINR constraints in (8) can be always made feasible. We use the notation a to denote a vector obtained by stacking $a_{b i}$ on top of each other, i.e., $\mathbf{a}=\left[a_{b 1}, a_{b 2}, \ldots, a_{b I}\right]$. In order to enable communication between primary and secondary vehicles, a set of suitable primary vehicles should be chosen. Selection of primary vehicles is done by solving an admission control problem.

Let $\hat{\mathcal{P}}$ denote the set of primary vehicles which are admitted. Maximizing the number of admitted vehicles that satisfy the SINR constraints is equivalent to minimizing the number of vehicles that requires a strictly positive value of $a_{b i}$ that satisfy constraint (8). Hence, by using above expressions the problem of joint admission control and association can be expressed as an optimization problem.

$$
\begin{aligned}
\operatorname{minimize} & \|\mathbf{a}\|_{0} \\
\text { subject to } & \Gamma_{b i} \geq \Gamma_{b i}^{t h}-a_{b i}, \quad \forall i \in \mathcal{P} \\
& a_{b i} \geq 0, \quad \forall i \in \mathcal{P} \\
& \sum_{i \in \mathcal{P}} P_{b i} \leq P_{b}^{\max }, \quad \forall i \in \mathcal{P} \\
& 0 \leq \sum_{i \in \hat{\mathcal{P}}} x_{i j} \leq 1, \quad \forall j \in \mathcal{S} \\
& \sum_{j \in \mathcal{S}} x_{i j} P_{i j} \leq P_{i}^{\max }, \quad \forall i \in \mathcal{P} \\
& \Gamma_{i j} \geq \Gamma_{i j}^{t h}, \quad \forall j \in \mathcal{S}
\end{aligned}
$$

where the optimization variables are $a_{b i}$ for $i \in \mathcal{P}$ and $x_{i j}$ for $i \in \mathcal{P}, j \in \mathcal{S}$.

Constraints (9a) and (9b) ensure slack variable definition is not violated, and constraint $(9 \mathrm{c})$ is the maximum power constraint at the BS. Constraint (9d) is used to ensure that every secondary vehicle is associated only with an admitted primary vehicle and every secondary vehicle should be associated with only one primary vehicle. Constraint (9e) is the maximum power constraint at the BS. Constraint (9f) ensures that every for primary to secondary vehicle link SINR is greater than its threshold level.

\section{Algorithm DERIVATION}

Problem (9) is combinatorial and it is an NP-hard problem, since the objective function is an $\ell_{0}$ function [18]. Thus, the complexity of finding an optimal solution for the problem grows with the problem size. Therefore, in the sequel, we first approximate the problem (9) so that it becomes noncombinatorial. We adopt a natural approach of relaxing the objective function to $l_{1}$ norm. Hence, the approximated problem can be formulated as below.

$$
\begin{aligned}
\text { minimize } & \|\mathbf{a}\|_{1} \\
\text { subject to } & \Gamma_{b i} \geq \Gamma_{b i}^{t h}-a_{b i}, \quad \forall i \in \mathcal{P} \\
& a_{b i} \geq 0, \quad \forall i \in \mathcal{P} \\
& \sum_{i \in \mathcal{P}} P_{b i} \leq P_{b}^{\max }, \quad \forall i \in \mathcal{P} \\
& 0 \leq \sum_{i \in \hat{\mathcal{P}}} x_{i j} \leq 1, \quad \forall j \in \mathcal{S} \\
& \sum_{j \in \mathcal{S}} x_{i j} P_{i j} \leq P_{i}^{\max }, \quad \forall i \in \mathcal{P} \\
& \Gamma_{i j} \geq \Gamma_{i j}^{t h}, \quad \forall j \in \mathcal{S}
\end{aligned}
$$

where $a_{b i}$ for $i \in \mathcal{P}$ and $x_{i j}$ for $i \in \mathcal{P}, j \in \mathcal{S}$ are the optimization variables.

Then we decompose the problem into two stages and propose a sub-optimal, two-stage algorithm to solve it. In the first stage of the algorithm we use admission control to select the best primary vehicles which can be admitted to the BS. In the second stage we associate secondary vehicles to the admitted primary vehicles from the first stage. The proposed algorithm is illustrated in Fig. 3. For admission control of primary vehicles, we solve the stage one of the problem by solving

$$
\begin{aligned}
\operatorname{minimize} & \|\mathbf{a}\|_{1} \\
\text { subject to } & \Gamma_{b i} \geq \Gamma_{b i}^{t h}-a_{b i}, \quad \forall i \in \mathcal{P} \\
& a_{b i} \geq 0, \quad \forall i \in \mathcal{P} \\
& \sum_{i \in \mathcal{P}} P_{b i} \leq P_{b}^{\text {max }}, \quad \forall i \in \mathcal{P},
\end{aligned}
$$

where the optimization variable is $a_{b i}$ for $i \in \mathcal{P}$.

When we obtain the optimal solution of the optimization problem (11), objective function of the problem (10) becomes a known vector and constraints (10a), (10b) and (10c) are satisfied. 
We define

$$
e_{b i}= \begin{cases}1, & \text { if } a_{b i}=0 \\ 0, & \text { otherwise }\end{cases}
$$

Admission control vector for the primary vehicles is defined by the optimal solution of the optimization problem (11). We define admission control vector $\mathbf{e}^{\star}$ by stacking $e_{b i}$ to a vector, where $\mathbf{e}^{\star}=\left[e_{b 1}, \ldots, e_{b I}\right]$ and each element represents whether the corresponding primary vehicle is admitted or not.

Next we utilize this to solve the second stage of the proposed algorithm. Hence, we can simplify problem (10) to the following feasibility problem

$$
\begin{aligned}
\text { minimize } & 0 \\
\text { subject to } & 0 \leq \sum_{i \in \hat{\mathcal{P}}} x_{i j} \leq 1, \quad \forall j \in \mathcal{S} \\
& \sum_{j \in \mathcal{S}} x_{i j} P_{i j} \leq P_{i}^{\max }, \quad \forall i \in \mathcal{P} \\
& \Gamma_{i j} \geq \Gamma_{i j}^{t h}, \quad \forall j \in \mathcal{S},
\end{aligned}
$$

where the optimization variable is $x_{i j}$ for $i \in \mathcal{P}, j \in \mathcal{S}$.

Finally, we summarize the proposed algorithm by Algorithm 1 given below.
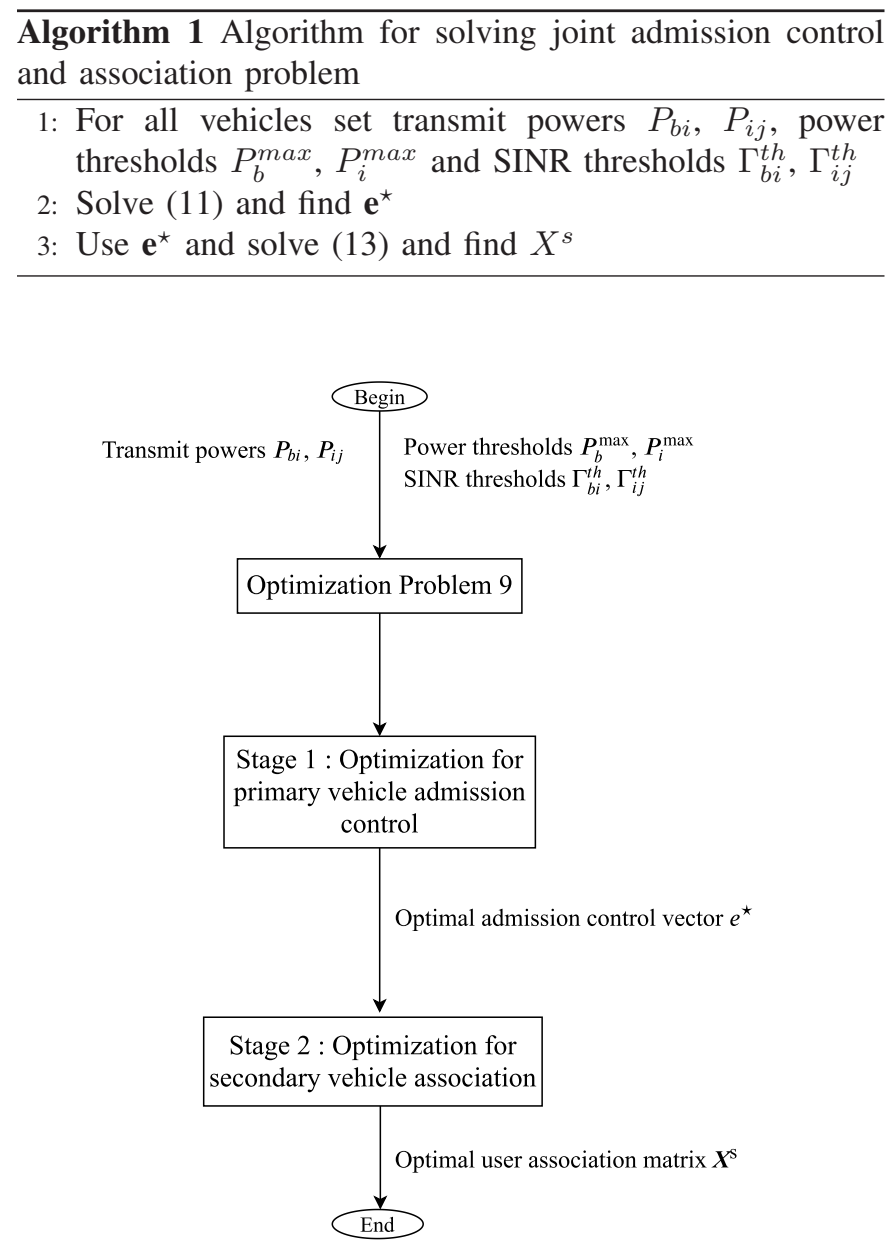

Fig. 3. Proposed two-stage algorithm

\section{Numerical Results}

In this section, we evaluate the performance of the proposed algorithm 1 with numerical simulations. It is assumed alignment procedure for all links have been completed. Therefore the alignment error between transmitter and receiver for all links from base station to primary vehicles and primary vehicles to secondary vehicles is considered to be zero.

To implement proposed algorithm we used CVX, a package for specifying and solving convex programs [19] and MOSEK solver has been used. Key parameters used for the simulations are given in Table I.

TABLE I

SIMULATION PARAMETERS

\begin{tabular}{|c|c|}
\hline Parameter & Value \\
\hline Half power beamwidth $\varphi$ & $\pi / 6$ \\
\hline Side lobe gain $\mathrm{z}$ & 0.1 \\
\hline Path loss exponent $\delta_{b i}$ and $\delta_{i j}$ & 2.1 \\
\hline$\beta_{b i}$ and $\beta_{i j}$ & 75.1 \\
\hline Maximum transmit power of base station $P_{b}^{\text {max }}$ & $40 \mathrm{dBm}$ \\
\hline Maximum transmit power of primary vehicle $P_{i}^{\text {max }}$ & $15 \mathrm{dBm}$ \\
\hline Thermal noise density $N_{0}$ & $-174 \mathrm{dBm} / \mathrm{Hz}$ \\
\hline Carrier frequency & $60 \mathrm{GHz}$ \\
\hline
\end{tabular}

Distance between two nodes $i$ and $j$ are defined by $d_{i j}$, maximum transmitting distance of $b$ is denoted by $d_{b}^{\max }$ and maximum transmitting distance of a primary vehicle is denoted by $d_{i}^{\max }$. To simplify the simulation setup, we define set of primary and secondary vehicles as follows.

$$
\begin{aligned}
& \mathcal{P}=\left\{i \mid d_{b i} \leq d_{b}^{\max }, i \in \mathcal{V}\right\}, \\
& \mathcal{S}=\left\{j \mid d_{i j} \leq d_{i}^{\text {max }}, j \in \mathcal{V}\right\} .
\end{aligned}
$$

In our simulations, we initialize all the vehicle positions randomly. Distances between BS and primary vehicles and primary to secondary vehicles vary in the range of 10 to $500 \mathrm{~m}$. We refer to arbitrarily generated set of topologies denoted by

$$
\mathcal{T}_{k}=\left\{d_{b i}, d_{i j} \mid \forall i \in \mathcal{P}, \forall j \in \mathcal{S}\right\},
$$

as a single topology realization.

As the baseline algorithm for comparing the stage-one of the proposed algorithm, exhaustive search has been used (since it can find the optimum solution for the problem). Exhaustive search algorithm finds the maximum number of primary vehicles that can be admitted by checking all possible combinations with the given constraints. Topologies are selected according to the set defined in (16) and we use 10 primary vehicles for stage-one simulations.

In order to provide a statistical description of number of admissions of the algorithm, we consider the cumulative distribution function (CDF) plots for number of admitted primary vehicles using the proposed algorithm and exhaustive search as shown in Fig. 4 for two different SINR threshold values. We have computed this simulation over 1000 topology realizations. It can be observed that exhaustive search and proposed algorithm show near equal performance. Hence, the proposed algorithm is able to find the maximum number of 


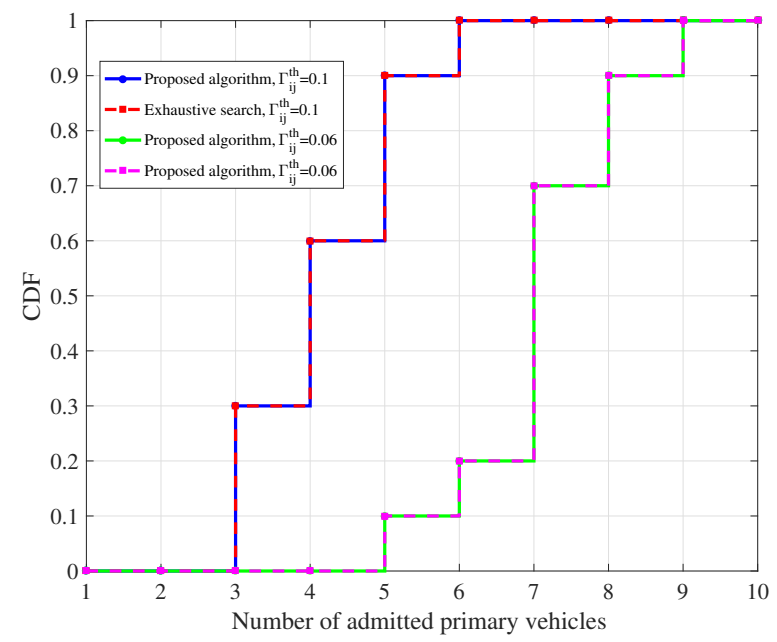

Fig. 4. CDFs for number of admitted primary vehicles

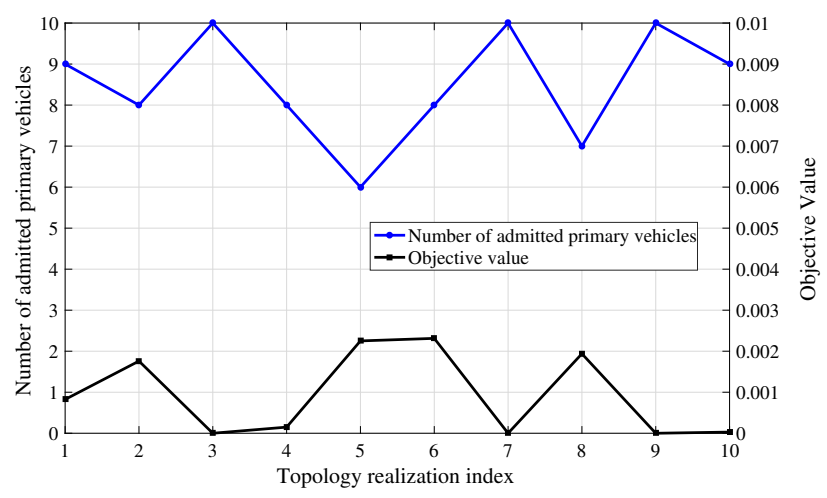

Fig. 5. Number of admitted primary vehicles and objective value vs topology realizations

admitted primary vehicles. In Fig. 5 we evaluate the variation of number of admitted primary vehicles and the objective value of the algorithm for ten topology realizations. We observe that when the objective value reaches the minimum value zero, maximum number of admitted primary vehicles, i.e., number ten is reached.

We can observe the average number of admitted vehicles is nearly equal for both algorithms by referring to Fig. 6 . The obtained results have been computed over 1000 topology realizations. When the SINR threshold is increased the number of vehicles which can satisfy the requirement decreases. Also this graph confirms that with the varying SINR target value, our proposed algorithm shows a closely similar performance.

To evaluate the performance of the stage two of the proposed algorithm, we consider a system of five primary vehicles and ten secondary vehicles. We evaluate the number of secondary vehicle associations with the target number of secondary vehicle associations. According to the algorithm if the constraints are satisfied, all secondary vehicles should associate with the set of admitted primary vehicles. From Fig. 7 it can be observed that number of secondary vehicle associ-

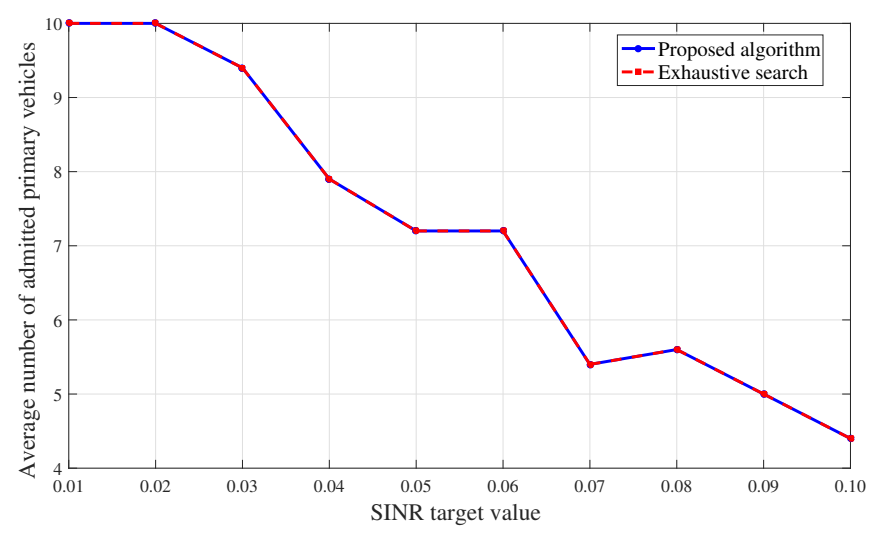

Fig. 6. Average number of admitted vehicles vs SINR target

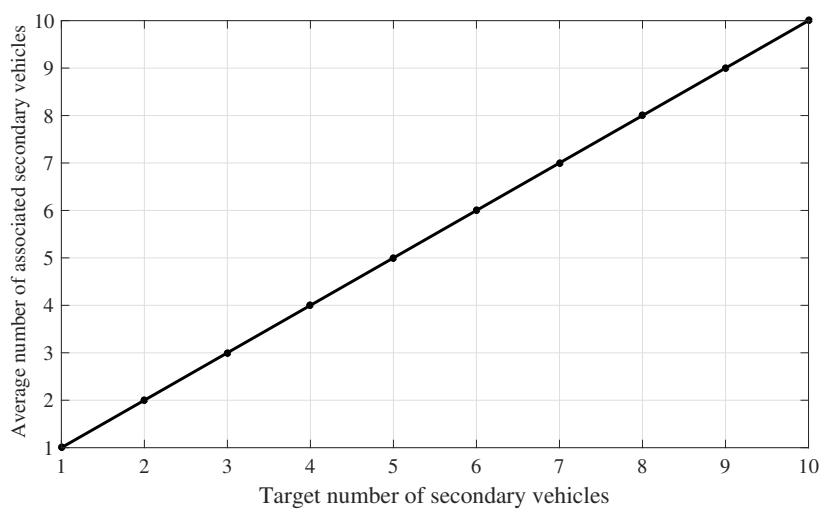

Fig. 7. Number of associated secondary vehicles vs target number of secondary vehicles.

ations are always equal to target number of associations. We have computed over 2000 topology realizations for obtained results. Therefore it can be seen that stage two of the proposed algorithm gives expected performance.

\section{Conclusions}

In this paper, we have considered the joint admission and association problem for a mmWave V2X network. We cast this problem as an $\ell_{0}$ minimization problem, and it is known to be NP-hard. Thus, we have proposed a two-stage algorithm to find a sub-optimal solution for this problem. First we solve the optimal admission control for the primary vehicles and secondly associate secondary vehicles to admitted primary vehicles. Numerically, we have shown that the stage-one of the proposed algorithm performs closely to exhaustive each algorithm, and stage-two of the algorithm associate all the secondary users while satisfying the system constraints. Thus, our proposed algorithm can be utilized to determine the optimal topology to provide connectivity for out of coverage vehicles from base station in practical VANETs. This research work can be extended further considering dynamic bandwidth allocation. 


\section{REFERENCES}

[1] M. Boban, A. Kousaridas, K. Manolakis, J. Eichinger, and W. Xu, "Connected roads of the future: Use cases, requirements, and design considerations for vehicle-to-everything communications," IEEE Vehicular Technology Magazine, vol. 13, no. 3, pp. 110-123, Sep. 2018.

[2] Z. MacHardy, A. Khan, K. Obana, and S. Iwashina, "V2X access technologies: Regulation, research, and remaining challenges," IEEE Communications Surveys Tutorials, vol. 20, no. 3, pp. 1858-1877, third quarter 2018.

[3] R. Molina-Masegosa and J. Gozalvez, "LTE-V for sidelink 5G V2X vehicular communications: A new $5 \mathrm{G}$ technology for short-range vehicleto-everything communications," IEEE Vehicular Technology Magazine, vol. 12 , no. 4, pp. 30-39, Dec. 2017.

[4] 3rd Generation Partnership Project (3GPP); Technical Specification Group Radio Access Network, “ TS 36.213 Evolved Universal Terrestrial Radio Access (E- UTRA); Physical layer procedures (v14.8.0, Release 14)," 3rd Generation Partnership Project (3GPP), Tech. Rep., 2017.

[5] M. J. Booysen, S. Zeadally, and G. . van Rooyen, "Survey of media access control protocols for vehicular ad hoc networks," IET Соттиnications, vol. 5, no. 11, pp. 1619-1631, Jul. 2011.

[6] Y. Jeong, J. W. Chong, H. Shin, and M. Z. Win, "Intervehicle communication: Cox-fox modeling," IEEE Journal on Selected Areas in Communications, vol. 31, no. 9, pp. 418-433, Sep. 2013.

[7] K. A. Rahman and K. E. Tepe, "Towards a cross-layer based mac for smooth V2V and V2I communications for safety applications in dsrc/wave based systems," in 2014 IEEE Intelligent Vehicles Symposium Proceedings. IEEE, Jun. 2014, pp. 969-973.

[8] R. Zhang, X. Cheng, Q. Yao, C.-X. Wang, Y. Yang, and B. Jiao, "Interference graph-based resource-sharing schemes for vehicular networks," IEEE transactions on vehicular technology, vol. 62 , no. 8, pp. 4028 4039, Oct. 2013.

[9] M. Bengtsson and B. Ottersten, "Optimal and suboptimal transmit beamforming," Handbook of Antennas in Wireless Communications, Jan. 2001.

[10] R. Stridh, M. Bengtsson, and B. Ottersten, "System evaluation of optimal downlink beamforming with congestion control in wireless com- munication," IEEE Transactions on Wireless Communications, vol. 5, pp. 743 - 751, May. 2006

[11] K. S. Manosha, S. K. Joshi, M. Codreanu, N. Rajatheva, and M. Latvaaho, "Admission control algorithms for QoS-constrained multicell MISO downlink systems," IEEE Transactions on Wireless Communications, vol. 17, no. 3, pp. 1982-1999, Dec. 2017.

[12] W. Sun, D. Yuan, E. G. Ström, and F. Brännström, "Resource sharing and power allocation for D2D-based safety-critical V2X communications," in 2015 IEEE International Conference on Communication Workshop (ICCW), June 2015, pp. 2399-2405.

[13] S. Zhang, Y. Hou, X. Xu, and X. Tao, "Resource allocation in D2Dbased $\mathrm{V} 2 \mathrm{~V}$ communication for maximizing the number of concurrent transmissions," in 2016 IEEE 27th Annual International Symposium on Personal, Indoor, and Mobile Radio Communications (PIMRC), Sep. 2016, pp. 1-6.

[14] F. Khan and Z. Pi, "mmWave mobile broadband (MMB): Unleashing the 3-300GHz spectrum," in 34th IEEE Sarnoff Symposium, May. 2011, pp. 1-6.

[15] Y. Niu, Y. Li, D. Jin, L. Su, and A. Vasilakos, "A survey of millimeter wave (mmWave) communications for 5G: Opportunities and challenges," Wireless Networks, vol. 21, Feb. 2015.

[16] A. Yamamoto, K. Ogawa, T. Horimatsu, A. Kato, and M. Fujise, "Pathloss prediction models for intervehicle communication at $60 \mathrm{GHz}$," IEEE Transactions on Vehicular Technology, vol. 57, no. 1, pp. 6578, Jan. 2008

[17] J. Wildman, P. H. J. Nardelli, M. Latva-aho, and S. Weber, "On the joint impact of beamwidth and orientation error on throughput in directional wireless poisson networks," IEEE Transactions on Wireless Communications, vol. 13, no. 12, pp. 7072-7085, Dec. 2014.

[18] S. Boyd, " $\ell_{1}$-norm methods for convex-cardinality problems," 2007 , [Online]. Available: http://www.stanford.edu/class/ee364b/lectures/11_ slides.pdf.

[19] M. Grant and S. Boyd, "CVX: Matlab software for disciplined convex programming, version 2.1," http://cvxr.com/cvx, Mar. 2014. 